\title{
Microwave Attenuation and Prediction of Rain Outage for Wireless Networks in Pakistan's Tropical Region
}

\author{
Uzma Siddique, Laeeq Ahmad, and Gulistan Raja \\ Department of Electrical \& Electronic Engineering, University of Engineering \& Technology, Taxila 47050, Pakistan \\ Correspondence should be addressed to Uzma Siddique, uzma1776@gmail.com
}

Received 2 September 2010; Revised 16 December 2010; Accepted 11 January 2011

Academic Editor: Beatriz Ortega

Copyright () 2011 Uzma Siddique et al. This is an open access article distributed under the Creative Commons Attribution License, which permits unrestricted use, distribution, and reproduction in any medium, provided the original work is properly cited.

The microwave attenuation due to rainfall in tropical regions has not been very widely studied yet. In Pakistan's tropical environment, line-of-sight microwave communication links were set up and have been operated for several years to study the microwave attenuation characteristics due to tropical rainfall. In this paper the experimental results are presented, including the cumulative distributions of microwave attenuation and the relationship between specific attenuation and rainfall rate. In addition, a rain outage prediction model is proposed which not only predicts microwave radio link performance but will also be useful in calculating the link degradations due to interference issues. The main focus of this research work has been done keeping in mind the wireless networks of Pakistan. The results show that the rainfall rate, the microwave propagation characteristics, and outage predictions in Pakistan differ from the International Radio Consultative Committee predictions and ITU recommendation P530.7/8, respectively.

\section{Introduction}

Mobile operators are deploying increasingly complex wireless networks, and they need to optimize network performance to attract customers, reduce complexity, and get the most out of their network investment.

To be successful, mobile operators must maximize transmissions network capacity and performance during the initial network design and throughout the life of the network by addressing all the factors that can affect network performance such as, terrain, irregular network layout, traffic distribution, new service offers, and an increasing subscriber base. Existing GSM transmissions networks in Pakistan suffer from a number of issues including reliability, performance due to usage of inaccurate rain statistics, and lack of offering the desired quality of services to their mobile subscribers.

To study the microwave propagation characteristics, experiments have been carried out in Pakistan. Previously published articles have introduced the setup of the experiment, analysed the microwave attenuation due to rainfall at $21.225 \mathrm{GHz}$, proposed the cumulative distributions of attenuation and attenuation duration at frequencies of 15 and $38.6 \mathrm{GHz}$, and also obtained the frequency scaling empirical formulae. The experimental results obtained in Pakistan differ from the CCIR predictions [1].

The aims and objectives of this paper are to provide correct rain statistics for Pakistan region, impact of rain statistics on communication, and cumulative distribution of microwave attenuation and finally to propose a solution in order to predict the actual outage for transmission networks in Pakistan based on ITU recommendations [2].

The next sections will cover the impact of rain on transmission communication, rainfall rate, attenuation cumulative distribution and its comparison with CCIR recommendations, ITU outage prediction model, and finally the comparison of experimental results with ITU standard model [3] and actual outage prediction for Pakistan region. Conclusions are drawn in the last section.

\section{Impact of Rain on Wireless Communication}

Rainfall causes the severe degradation of the receive signal level above $10 \mathrm{GHz}$, and generally this degradation is directly proportional to the frequency of radio waves. Each particular raindrop contributes to the attenuation of the wanted signal. 


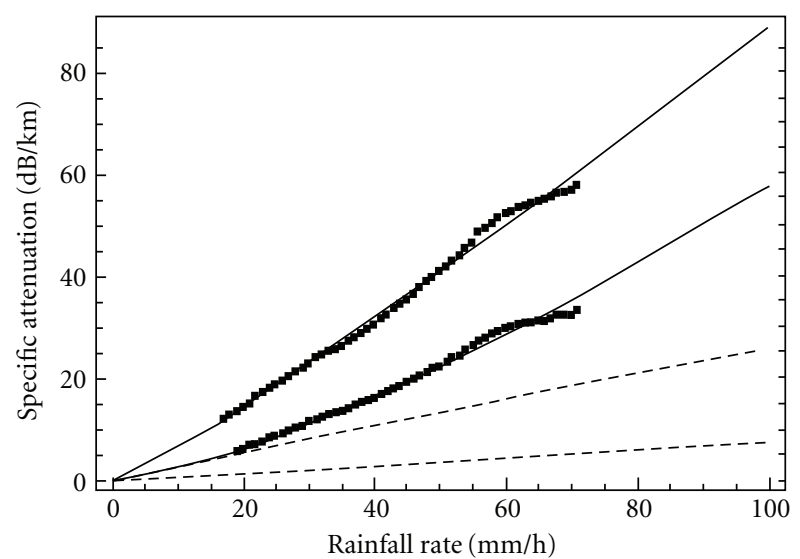

FIGURE 1: Relationship between rainfall rate and attenuation at frequencies of 15 and $38.6 \mathrm{GHz}$ [4].

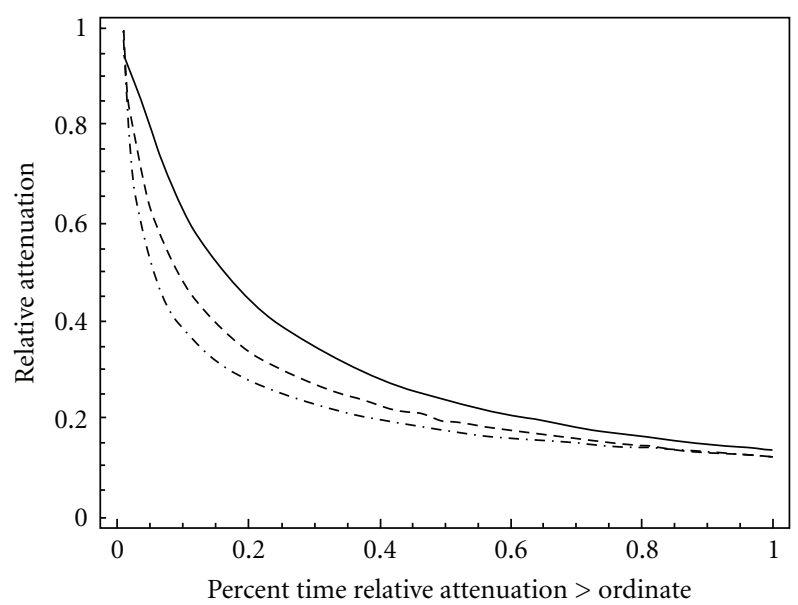

FIgURE 2: Comparison of cumulative attenuation distribution. The solid curve is from our measured results of 15 and $38.6 \mathrm{GHz}$, the dashed curve is from $21.225 \mathrm{GHz}$ given by [5], and the dash dotted curve is from [1].

The actual amount of fading is dependent on the frequency of the signal and the size of the raindrop. The two main causes of rain fading are scattering and absorption.

In both satellite and terrestrial communication applications the prediction of rainfall attenuation is requisite. In practice, the relationship between specific attenuation $A$ $(\mathrm{dB} / \mathrm{km})$ and rainfall rate $R(\mathrm{~mm} / \mathrm{hr})$ can be approximated by the power law

$$
A=a R^{b}
$$

where coefficients " $a$ " and " $b$ " depend on a variety of parameters (such as raindrop size distribution, raindrop shape, temperature, operating frequency, and polarization to a certainty) [4].

ITU has defined the entire Pakistan in $k$-rain region with $42 \mathrm{~mm} / \mathrm{hr}$ rain intensity. Within Pakistan there is a great variation in rain intensity from one city to another due to which different cities are under the influence of different regions ranging from $B$-rain zone to $P$-rain zone.
TABLE 1: ITU climatic zones [2].

\begin{tabular}{llccccccc}
\hline S. no & $\begin{array}{l}\text { percent age } \\
\text { of time } \\
\text { rain regions }\end{array}$ & 1 & 0.3 & 0.1 & 0.03 & 0.01 & 0.003 & 0.001 \\
\hline 1 & $A$ & 0.12 & 0.8 & 2 & 5 & 8 & 14 & 22 \\
2 & $B$ & 0.5 & 2 & 3 & 6 & 12 & 21 & 32 \\
3 & $C$ & 0.7 & 2.8 & 5 & 9 & 15 & 26 & 42 \\
4 & $D$ & 2.1 & 4.5 & 8 & 13 & 19 & 29 & 42 \\
5 & $E$ & 0.6 & 2.4 & 6 & 12 & 22 & 41 & 70 \\
6 & $F$ & 1.7 & 4.5 & 8 & 15 & 28 & 54 & 78 \\
7 & $G$ & 3 & 7 & 12 & 20 & 30 & 45 & 65 \\
8 & $H$ & 2 & 4 & 10 & 18 & 32 & 55 & 83 \\
9 & $J$ & 8 & 13 & 20 & 28 & 35 & 45 & 55 \\
$\mathbf{1 0}$ & K & $\mathbf{1 . 5}$ & $\mathbf{4 . 2}$ & $\mathbf{1 2}$ & $\mathbf{2 3}$ & $\mathbf{4 2}$ & $\mathbf{7 0}$ & $\mathbf{1 0 0}$ \\
11 & $L$ & 2 & 7 & 15 & 33 & 60 & 105 & 150 \\
12 & $M$ & 4 & 11 & 22 & 40 & 63 & 95 & 120 \\
13 & $N$ & 5 & 15 & 35 & 65 & 95 & 140 & 180 \\
14 & $P$ & 12 & 34 & 65 & 105 & 145 & 200 & 250 \\
15 & $Q$ & 24 & 49 & 72 & 96 & 115 & 142 & 170 \\
\hline
\end{tabular}

Karachi has $148 \mathrm{~mm} / \mathrm{hr}(P$-region) rain intensity, recorded in August 2007. Islamabad is under $L$-region $(60 \mathrm{~mm} / \mathrm{hr})$, while Peshawar and Faisalabad come after $K$-region with $42 \mathrm{~mm} / \mathrm{hr}$ rain intensity. However, the regions like Thar and Cholistan Desert are in the continuous state of drought for the last 5-6 years.

In a nutshell, Pakistan can be divided into various rain zones, and therefore every rain region has different effect on microwave propagation.

\section{Impact of Using Wrong Rain Statistics on Wireless Communication}

For areas with rain region less than " $K$ " planning with higher rain region increases the operator's OpEx. For example, in order to achieve $99.999 \%$ availability, for a given path length, a single microwave hop can cater the requirement but planning the same link with wrong higher rain region will require more than one microwave hop to achieve the same availability objectives.

Similarly for areas with rain regions higher than $K$, planning with lower rain rate results in the poor performance of the transmission networks. For example, Karachi $(P$ region) when planned with $K$-region will suffer more outages than expected.

\section{ITU Outage Prediction Model}

ITU has divided the world in different rain climatic zones based on the rain intensity as shown in Table 1 .

A-region is with minimum rain intensity while $N$-Region is with maximum rain intensity. As we move towards higher rain regions microwave propagation is more affected by the rain. Rain outage time is calculated using the following steps. 


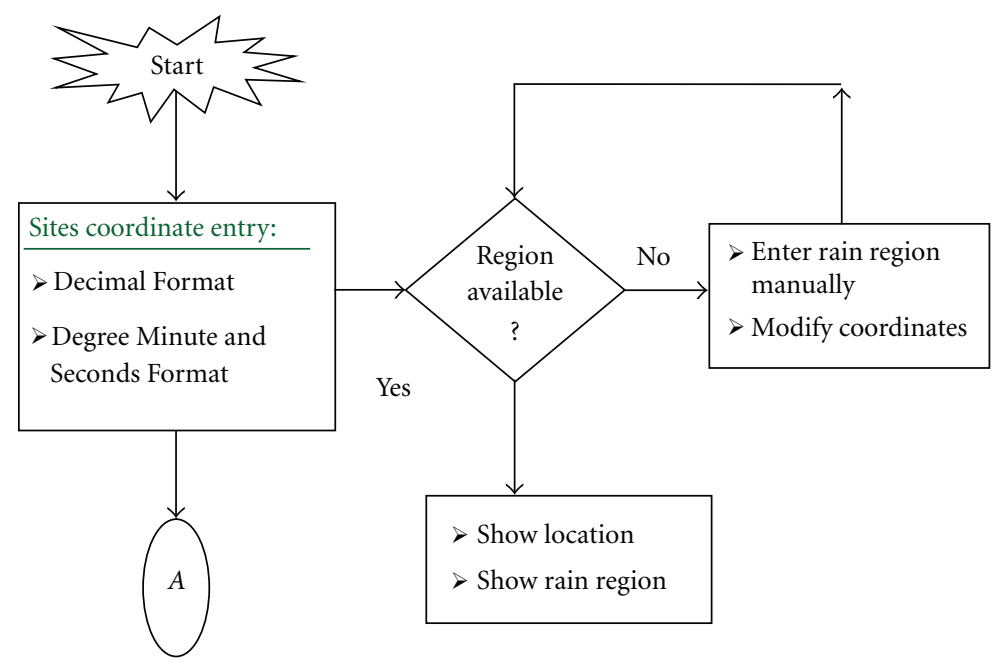

FIgURE 3: Processing step 1.

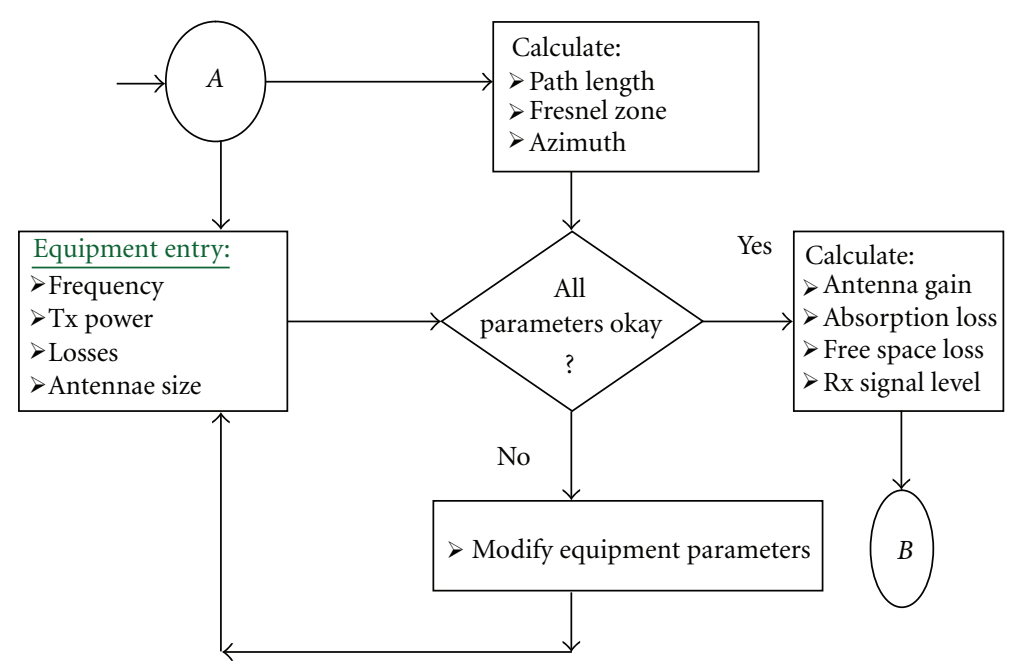

Figure 4: Processing step 2.

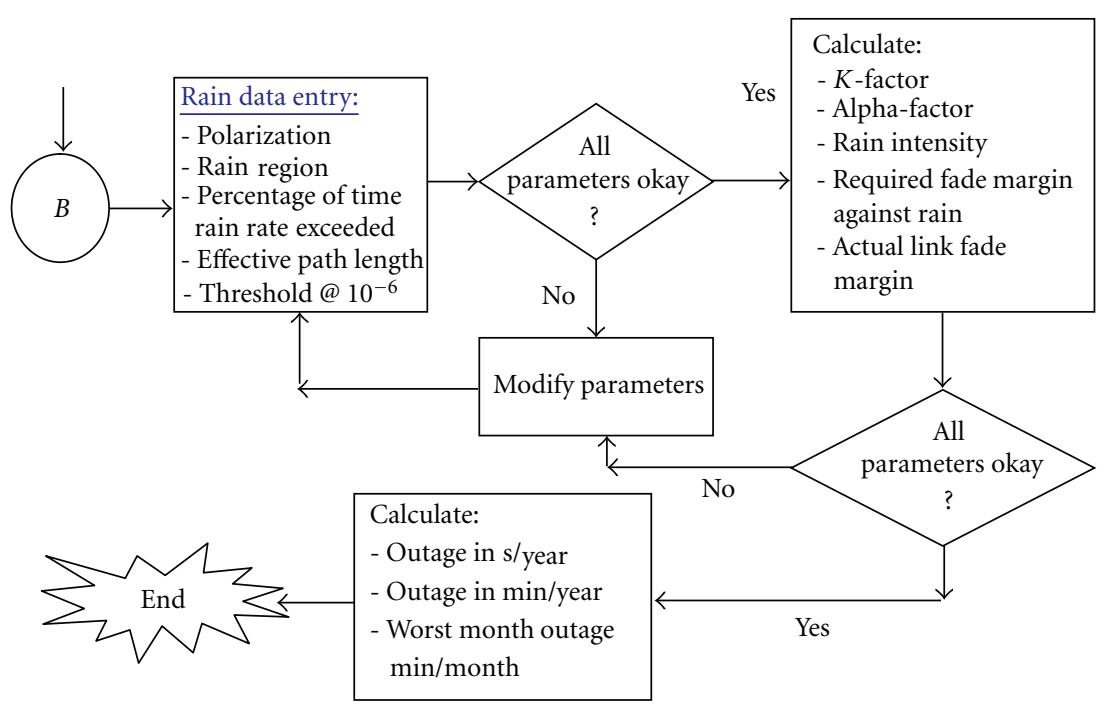

Figure 5: Processing step 3. 


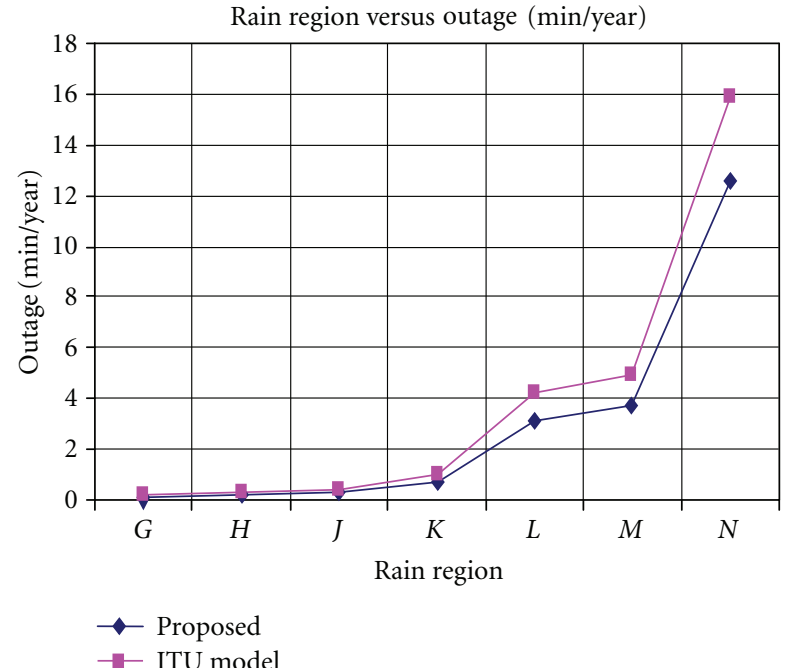

Figure 6: Comparison between Pathloss prediction model and proposed solution with respect to rain Region.

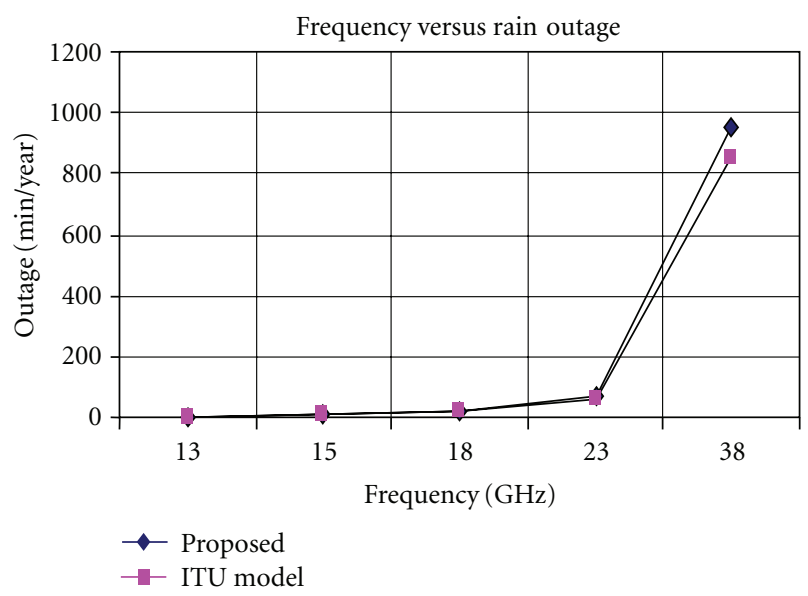

Figure 7: Comparison between Pathloss prediction model and proposed solution with respect to frequency.

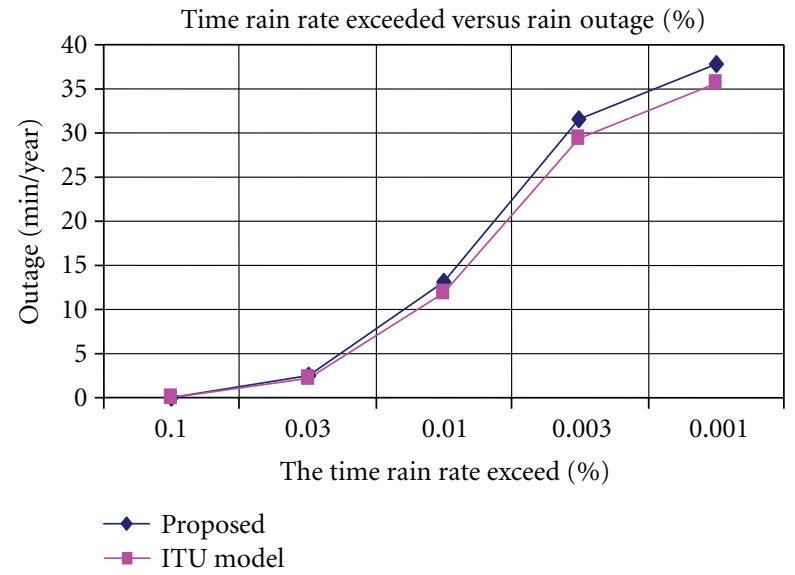

Figure 8: Comparison between Pathloss prediction model and proposed with respect to percentage of time rain rate exceeded.
Determine the rain rate, $R_{0.01}$, exceeded for $0.01 \%$ of the time [2].

Calculate the specific attenuation " $\gamma$ " [2], where

$$
\gamma=\alpha_{\alpha}^{\alpha} R_{0.01 \%}^{\beta}
$$

where $\boldsymbol{\alpha}$ and $\boldsymbol{\beta}$ are regression coefficients.

Effective path length is calculated as follows [2]:

$$
\begin{gathered}
d_{e}=\frac{d}{\left(1+d / d_{o}\right)}, \\
d_{o}=34 \cdot e^{-\left(0.15 R_{0.01}\right)} .
\end{gathered}
$$

The path attenuation, $A_{0.01 \%}$, exceeded for $0.01 \%$ of the time, is given by [2]

$$
A_{0.01 \%}=\gamma \cdot d_{e}
$$

Attenuations, $A$, exceeded for other percentages of the time, $P$, are derived from [2]

$$
\frac{A}{A_{0.01 \%}}=0.12 P^{-[0.546+0.043 \cdot \log 10(P)]} .
$$

The above equation is valid for latitude equal to or greater than 30 degree North or South [2]:

$$
\frac{A}{A_{0.01 \%}}=0.12 P^{-[0.855+0.139 \cdot \log 10(P)]} .
$$

Above equation is valid for latitude less than 30 degree North or South. The attenuation $A$ is set to the path fade margin and the equation is solved for $P$.

The ITU 530 model [2] clearly states that these calculations are valid for $1 \%$ to $0.001 \%$ of the time rain rate exceeded.

The worst month outage can be calculated by using the given formula [2]:

$$
T_{\mathrm{wm}}=1.22 T_{\mathrm{an}}^{(0.87)},
$$

where $T_{\mathrm{wm}}$ is the worst month outage in seconds, and $T_{\mathrm{an}}$ is annual outage time in second.

\section{Attenuation Cumulative Distribution}

We have experimentally studied the microwave propagation characteristics at frequencies of 15 and $38.6 \mathrm{GHz}$. There exists an almost linear relationship between specific attenuation $A$ (in decibels) and the logarithmic value of the percentage time $F$ (in percent); for $15 \mathrm{GHz}$ [6],

$$
A_{15}=1.415-15.66 \log (F),
$$

while, for $38.6 \mathrm{GHz}$,

$$
A_{38.6}=7.182-24.93 \log (F) .
$$

Both the correlative coefficients between $A$ and $\log (F)$ are almost -1 for the percentage time $F$ within the range from $0.5 \%$ to $0.01 \%$. 
Adopting the widely used formula type $A / A_{0.01}=$ $c F^{-[a+b \log (F)]}(a, b$, and $c$ are constant coefficients) [1], we derive the formulae as follows:

$$
\frac{A_{15}}{A_{0.01}}=0.097 F^{-[1.096+0.305 \log (F)]},
$$

where $A_{0.01}$ for $15 \mathrm{GHz}$ is $29.5 \mathrm{~dB}$,

$$
\frac{A_{38.6}}{A_{0.01}}=0.156 F^{-[0.821+0.215 \log (F)]},
$$

where $A_{0.01}$ for $38.6 \mathrm{GHz}$ is $52.5 \mathrm{~dB}$.

The CCIR prediction [1] provides the formula as follows:

$$
\frac{A}{A_{0.01}}=0.12 F^{-[0.546+0.043 \log (F)]}
$$

and the result for $21.225 \mathrm{GHz}$ given previously by Yeo et al. $[5]$ is

$$
\frac{A}{A_{0.01}}=0.12 F^{-[0.74+0.14 \log (F)]} .
$$

To make a good comparison, three curves have been drawn in Figure 2 using these formulae. The three curves stand for (10), (12), and (11), respectively, from top to bottom.

From Figure 2 the following points are noted.

(1) According to $[5,6]$, CCIR prediction has underestimated the specific attenuation level for a specific percentage time between $0.01 \%$ and $0.5 \%$ in tropical regions like Pakistan.

(2) The coefficient b for the Pakistan's tropical region is bigger than that of [1]. Since coefficient $b$ is of almost the same order as coefficient $a$ in our experiment, this type of formula is quite fallible for the small percentage time (e.g., smaller than $0.001 \%$ ).

\section{Proposed Outage Prediction Model}

The goal of the proposed outage prediction model is to predict radio relay link performance (quality and unavailability). The formulas are computed according to recommendation ITU-R P.530-7/8 [2]. This outage prediction model has the following main features:

(i) link outage calculation (min/year),

(ii) link outage for the rain rate exceeding all the percentages of time $(1 \%-0.001 \%)$,

(iii) worst month outage calculation.

Some facts and figures about proposed solutions are as follows.

(i) Due to unavailability of rain data for the entire Pakistan this model will simulate the calculations for only 5 cities, that is, Islamabad, Peshawar, Faisalabad, Lahore, and Multan.

(ii) The proposed solution is based on ITU recommendations P-530.7/8 [2]. (iii) Apart from annual outages, the proposed prediction model can calculate the worst month outage calculation also.

The first processing step is to enter the site coordinates either in Decimal Format or in Degree Minute and Seconds Format. On the basis of the given input the city and its rain region is identified by the model. If the given inputs are outside the database, the model will simply return an invalid result and then either modify the coordinates or manually enter the rain zone for the given coordinates.

In the second step, Path Profile Generator generates the path length, Fresnel zone radius, and azimuth based on the coordinates of the two sites. Then Logical Tester verifies the validity of the given parameters. If all parameters are okay, then Gain and Loss Analyzer generate antennas gain, absorption losses, free space loss, and Rx signal level. In case of any invalid entry the parameters like frequency, transmit power, losses, and antenna size should be modified.

The final step includes the entry of parameters related to rain, that is, polarization, rain region, percentage of time rain rate exceeded, effective path length, and threshold @ $10^{-6}$. These parameters are first verified for their validity.

If all parameters are okay the output results are displayed in terms of $K$-Factor, alpha factor, rain intensity, required fade margin against rain, and actual link fade margin.

On the basis of above input the final calculations are done to get the outages in sec/year, outage in min/year, and worst month outage in $\mathrm{min} / \mathrm{month}$.

\section{Comparison between the Two Models}

The comparison between Pathloss prediction model [3] based on ITU recommendation P 530.7/8 [2] and the proposed solution is made with respect to different parameters.

The graph in Figure 6 represents the variation of outage, with respect to rain region keeping path length, frequency and polarization constant, when comparing the two models.

This variation in the results, calculated by the two models, is directly proportional to the rain intensity. The variation is small with lower rain region and increases as the rain intensity increases. In nutshell the averaged variation in outage prediction, computed by the two models, is around $0.2 \%$.

The graph in Figure 7 represents the variation of outage, with respect to frequency variation, keeping path length, rain region, and polarization constant, computed by the two different models.

The results are almost similar at lower and medium frequencies whereas at higher frequencies the variation in the result is about $0.05 \%$. At higher frequencies voice communication is more susceptible to propagation effects especially rain as compared to lower frequencies.

The graph in Figure 8 represents the variation of outage, with respect to percentage of time rain rate exceeded keeping path length, rain region, and polarization constant, when comparing the two models.

The results are almost similar at different values, with a slight variation of about $0.25 \%$. 


\section{Conclusions}

In Pakistan's tropical region, a line of sight microwave communication experiments have been conducted to study the microwave-specific attenuation due to rainfall at frequencies of 15 and $38.6 \mathrm{GHz}$.

This paper has presented the results on rainfall rate, attenuation cumulative distribution, the relationship between specific attenuation and rainfall rate, and rain outage prediction model.

ITU has defined the entire Pakistan in K-rain Region with $42 \mathrm{~mm} / \mathrm{hr}$ rain intensity. Rain intensity in Pakistan in 2007 has exceeded $30 \%$ as compared to previous years.

The proposed solution is supported by the two features that were not taken into account by Pathloss prediction model, extensively used by transmission planners, based on ITU recommendations P530.7/8 [2].

(i) The entire Pakistan is no more in single rain profile. Therefore the effect of rain intensity on microwave propagation is variable as different cities have been assigned variable rain regions.

(ii) The outage calculations can be computed for the rain rate exceeded other than $0.01 \%$ of the time.

Rain region is calculated according to the data collected from meteorological office and outages other than $0.01 \%$ of the time rain rate exceeded can be calculated.

The experimental results after comparison, between ITU standard model [2] and the proposed solution with respect to different parameters, show deviation of about $0.05 \%-$ $3 \%$. Apart from annual outages, the proposed solution can calculate the worst month outage also. The proposed solution can be used for mobile communication planners since it contains all the basic parameters for the transmission link planning.

It has been proposed that the raindrop model and raindrop size distribution in Pakistan are quite different from those adopted by CCIR, and this will be studied further.

\section{Acknowledgments}

The authors would like to thank the Meteorological Department of Pakistan for providing correct rain statistics of different regions of Pakistan. Finally, they would like to pay special thanks to Siemens Pakistan for provision of ITU standard software Pathloss.

\section{References}

[1] CCIR, "Radiometeorological data," Rep. 563-3, Int. Telecommun. Union, Geneva, Switzerland, 1986.

[2] ITU Recommendations P.530-7/8, "Propagation Data and Prediction Methods for Terrestrial Line of Sight Systems".

[3] ITU standard model PATHLOSS.

[4] Z. X. Zhou, L. W. Li, T. S. Yeo, and M. S. Leong, "Cumulative distributions of rainfall rate and microwave attenuation in Singapore's tropical region," Radio Science, vol. 35, no. 3, pp. 751-756, 2000 .
[5] T. S. Yeo, P. S. Kooi, and M. S. Leong, "Two-year measurement of rainfall attenuation of CW microwaves in Singapore," IEEE Transactions on Antennas and Propagation, vol. 41, no. 6, pp. 709-712, 1993.

[6] Z. X. Zhou, L. W. Li, T. S. Yeo, and M. S. Leong, "Analysis of experimental results on microwave propagation in Singapore's tropical rainfall environment," Microwave and Optical Technology Letters, vol. 21, no. 6, pp. 470-473, 1999. 

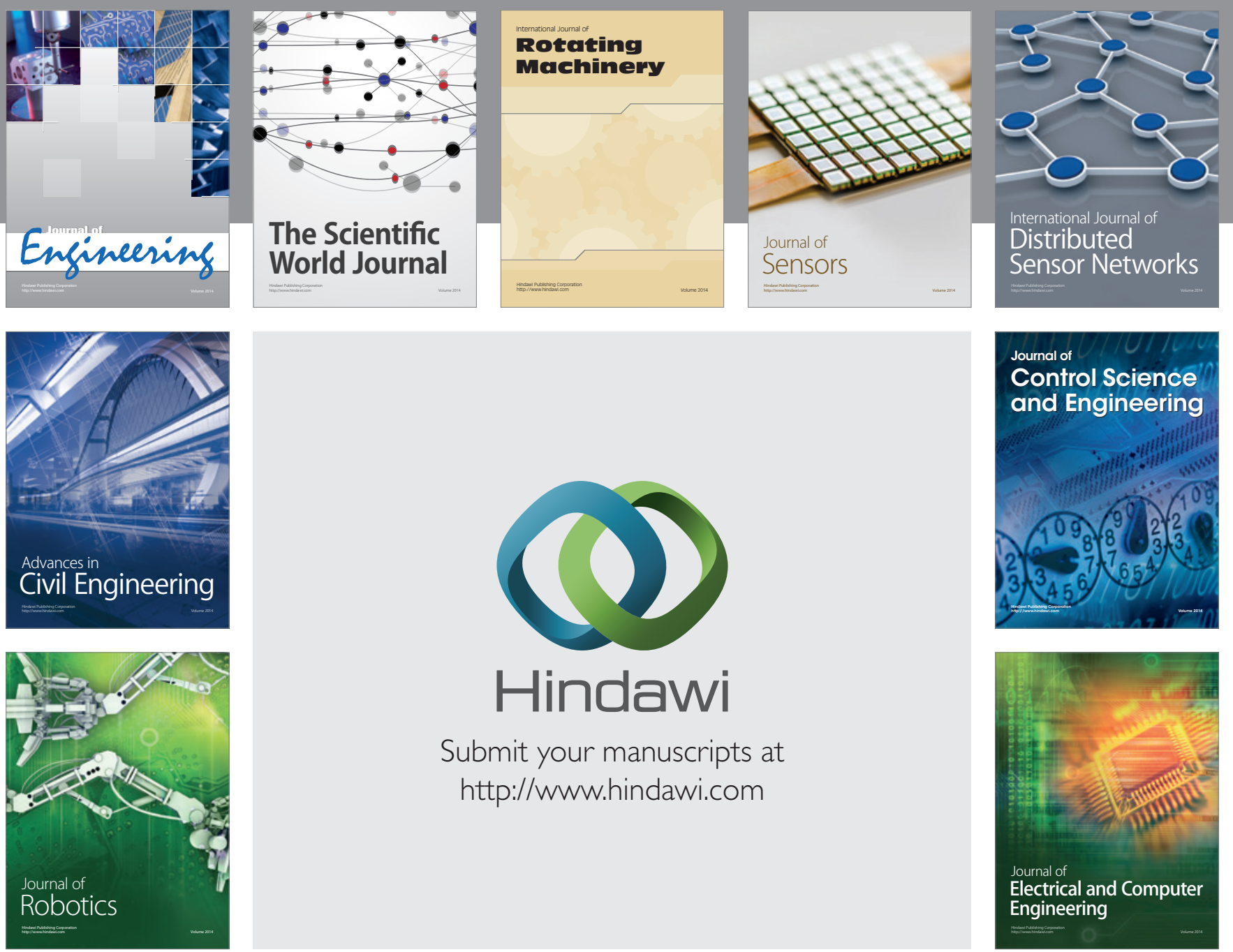

Submit your manuscripts at

http://www.hindawi.com
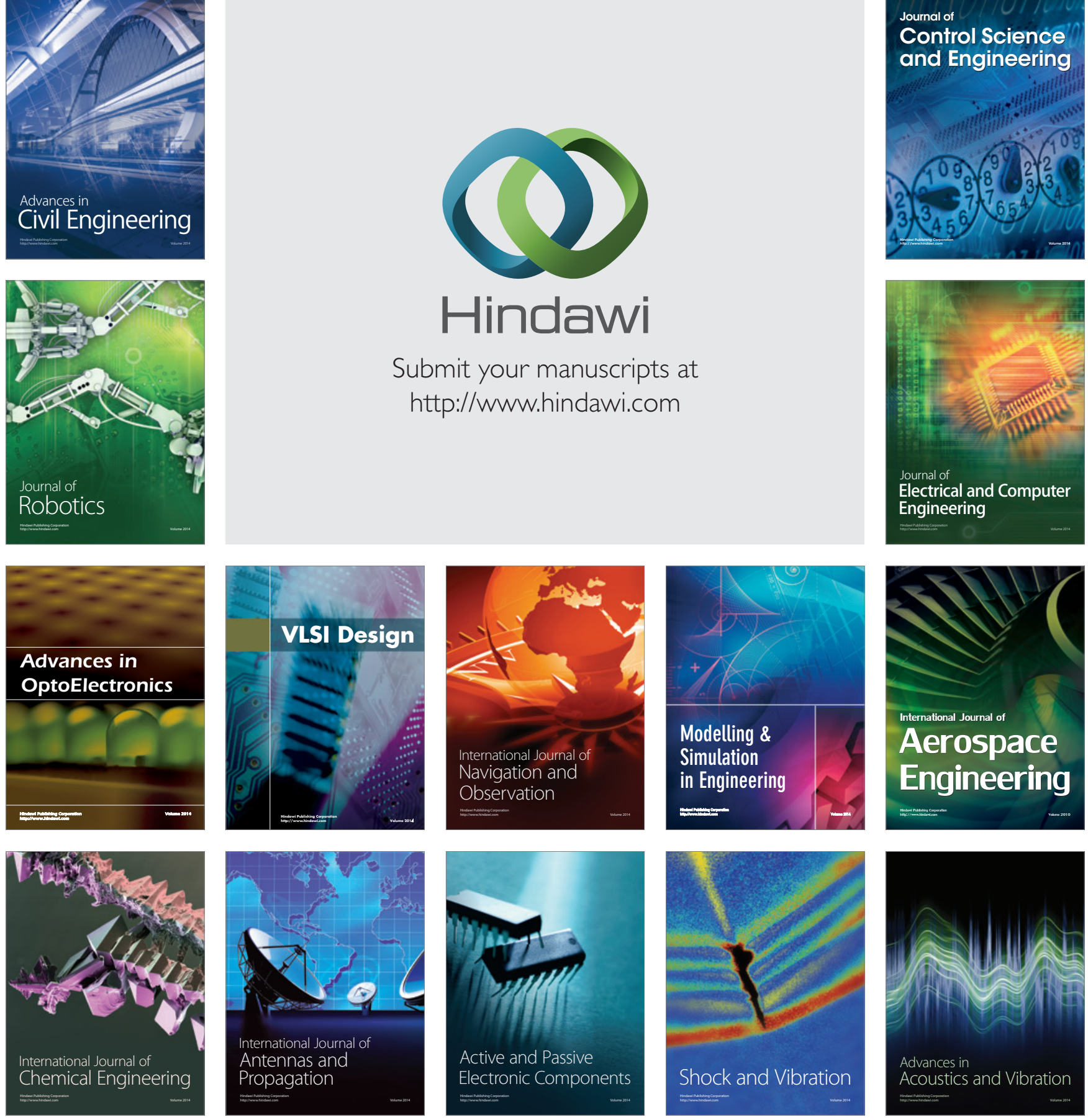